


\section{The Dibner Library of the History of}

Science and Technology

SMITHSONIAN INSTITUTION LIBRARIES

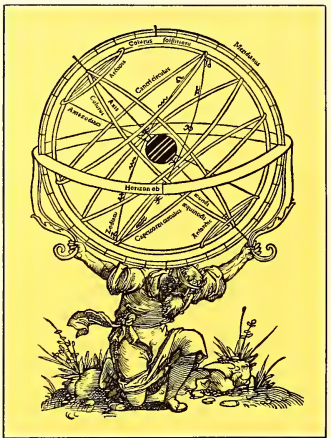






$$
\text { list }
$$



V. Experimental Researches in Electricity. By Michael Faraday, F.R.S., M.R.I., Corr. Mem. Royal Acad. of Sciences of Paris, Petersburgh, \& c. \& c.

Read November 24, 1831. .

\$1. On the Induction of Electric Currents. \$2. On the Evolution of Electricity from Magnetism. $§ 3$. On a new Electrical Condition of Matter. §4. On Arago's Magnetic Phenomena.

1. THE power which electricity of tension possesses of causing an opposite electrical state in its vicinity has been expressed by the general term Induction; which, as it has been received into scientific language, may also, with propriety, be used in the same general sense to express the power which electrical currents may possess of inducing any particular state upon matter in their immediate neighbourhood, otherwise indifferent. It is with this meaning that I purpose using it in the present paper.

2. Certain effects of the induction of electrical currents have already been recognised and described: as those of magnetization; AMPERE's experiments of bringing a copper disc near to a flat spiral; his repetition with electromagnets of Arago's extraordinary experiments, and perhaps a few others. Still it appeared unlikely that these could be all the effects induction by currents could produce; especially as, upon dispensing with iron, almost the whole of them disappear, whilst yet an infinity of bodies, exhibiting definite phenomena of induction with electricity of tension, still remain to be acted upon by the induction of electricity in motion.

3. Further: Whether Ampere's beautiful theory were adopted, or any other, or whatever reservation were mentally made, still it appeared very extraordinary, that as every electric current was accompanied by a corresponding intensity of magnetic action at right angles to the current, good conductors of electricity, when placed within the sphere of this action, should not have any 
current induced through them, or some sensible effect produced equivalent in force to such a current.

4. These considerations, with their consequence, the hope of obtaining electricity from ordinary magnetism, have stimulated me at various times to investigate experimentally the inductive effect of electric currents. I lately arrived at positive results; and not only had my hopes fulfilled, but obtained a key which appeared to me to open out a full explanation of Arago's magnetic phenomena, and also to discover a new state, which may probably have great influence in some of the most important effects of electric currents.

5. These results I purpose describing, not as they were obtained, but in such a manner as to give the most concise view of the whole.

\section{§. 1. Induction of Electric Currents.}

6. About twenty-six feet of copper wire one twentieth of an inch in diameter were wound round a cylinder of wood as a helix, the different spires of which were prevented from touching by a thin interposed twine. This helix was covered with calico, and then a second wire applied in the same manner. In this way twelve helices were superposed, each containing an average length of wire of twenty-seven feet, and all in the same direction. The first, third, fifth, seventh, ninth, and eleventh of these helices were connected at their extremities end to end, so as to form one helix; the others were connected in a similar manner; and thus two principal helices were produced, closely interposed, having the same direction, not touching anywhere, and each containing one hundred and fifty-five feet in length of wire.

7. One of these helices was connected with a galvanometer, the other with a voltaic battery of ten pairs of plates four inches square, with double coppers and well charged; yet not the slightest sensible deflection of the galvanometer needle could be observed.

8. A similar compound helix, consisting of six lengths of copper and six of soft iron wire, was constructed. The resulting iron helix contained two hundred and fourteen feet of wire, the resulting copper helix two hundred and eight feet; but whether the current from the trough was passed through the copper or the iron helix, no effect upon the other could be perceived at the galvanometer. 
9. In these and many other similar experiments no difference in action of any kind appeared between iron and other metals.

10. Two hundred and three feet of copper wire in one length were passed round a large block of wood; other two hundred and three feet of similar wire were interposed as a spiral between the turns of the first, and metallic contact everywhere prevented by twine. One of these helices was connected with a galvanometer, and the other with a battery of one hundred pairs of plates four inches square, with double coppers, and well charged. When the contact was made, there was a sudden and very slight effect at the galvanometer, and there was also a similar slight effect when the contact with the battery was broken. But whilst the voltaic current was continuing to pass through the one helix, no galvanometrical appearances of any effect like induction upon the other helix could be perceived, although the active power of the battery was proved to be great, by its heating the whole of its own helix, and by the brilliancy of the discharge when made through charcoal.

11. Repetition of the experiments with a battery of one hundred and twenty pairs of plates produced no other effects; but it was ascertained, both at this and the former time, that the slight deflection of the needle occurring at the moment of completing the connexion, was always in one direction, and that the equally slight deflection produced when the contact was broken, was in the other direction; and also, that these effects occurred when the first helices were used (6. 8.).

12. The results which $I$ had by this time obtained with magnets led me to believe that the battery current through one wire, did, in reality, induce a similar current through the other wire, but that it continued for an instant only, and partook more of the nature of the electrical wave passed through from the shock of a common Leyden jar than of that from a voltaic battery, and therefore might magnetise a steel needle, although it scarcely affected the galvanometer.

13. This expectation was confirmed; for on substituting a small hollow helix, formed round a glass tube, for the galvanometer, introducing a steel needle, making contact as before between the battery and the inducing wire (7. 10.), and then removing the needle before the battery contact was broken, it was found magnetised. 
14. When the battery contact was first made, then an unmagnetised needle introduced into the small indicating helix, and lastly the battery contact broken, the needle was found magnetised to an equal degree apparently with the first; but the poles were of the contrary kind.

15. The same effects took place on using the large compound helices first described (6. 8.).

16. When the unmagnetised needle was put into the indicating helix, before contact of the inducing wire with the battery, and remained there until the contact was broken, it exhibited little or no magnetism; the first effect having been nearly neutralised by the second (13.14.). The force of the induced current upon making contact was found always to exceed that of the induced current at breaking of contact; and if therefore the contact was made and broken many times in succession, whilst the needle remained in the indicating belix, it at last came out not unmagnetised, but a needle magnetised as if the induced current upon making contact had acted alone on it. This effect may be due to the accumulation (as it is called) at the poles of the unconnected pile, rendering the current upon first making contact more powerful than what it is afterwards, at the moment of breaking contact.

17. If the circuit between the helix or wire under induction and the galvanometer or indicating spiral was not rendered complete before the connexion between the battery and the inducing wire was completed or broken, then no effects were perceived at the galvanometer. Thus, if the battery communications were first made, and then the wire under induction connected with the indicating helix, no magnetising power was there exhibited. But still retaining the latter communications, when those with the battery were broken, a magnct was formed in the helix, but of the second kind, i. e. with poles indicating a current in the same direction to that belonging to the battery current, or to that always induced by that current in the first instance.

18. In the preceding experiments the wires were placed near to each other, and the contact of the inducing one with the battery made when the inductive effect was required; but as some particular action might be supposed to be exerted at the moments of making and breaking contact, the induction was produced in another way. Several feet of copper wire were stretched in wide zigzag forms, representing the letter $W$, on one surface of a broad board; a second 
wire was stretched in precisely similar forms on a second board, so that when brought near the first, the wires should everywhere touch, except that a sheet of thick paper was interposed. One of these wires was connected with the galvanometer, and the other with a voltaic battery. The first wire was then moved towards the second, and as it approached, the needle was deflected. Being then removed, the needle was deflected in the opposite direction. By first making the wires approach and then recede, simultaneously with the vibrations of the needle, the latter soon became very extensive; but when the wires ceased to move from or towards each other, the galvanometer needle soon came to its usual position.

19. As the wires approximated, the induced current was in the contrary direction to the inducing current. As the wires receded, the induced current was in the same direction as the inducing current. When the wires remained stationary, there was no induced current (54.).

20. When a small voltaic arrangement was introduced into the circuit between the galvanometer (10.) and its helix or wire, so as to cause a permanent deflection of $30^{\circ}$ or $40^{\circ}$, and then the battery of one hundred pairs of plates connected with the inducing wire, there was an instantaneous action as before (11.); but the galvanometer-needle immediately resumed and retained its place unaltered, notwithstanding the continued contact of the inducing wire with the trough : such was the case in whichever way the contacts were made (33.).

21. Hence it would appear that collateral currents, either in the same or in opposite directions, exert no permanent inducing power on each other, affecting their quantity or tension.

22. I could obtain no evidence by the tongue, by spark, or by heating fine wire or charcoal, of the electricity passing through the wire under induction; neither could I obtain any chemical effects, though the contacts with metallic and other solutions were made and broken alternately with those of the battery, so that the second effect of induction should not oppose or neutralize the first (13. 16.).

23. This deficiency of effect is not because the induced current of electricity cannot pass fluids, but probably because of its brief duration and feeble intensity; for on introducing two large copper plates into the circuit on the induced side (20.), the plates being immersed in brine, but prevented from 
touching each other by an interposed cloth, the effect at the indicating galvanometer, or helix, occurred as before. The induced electricity could also pass through the trough (20.). When, however, the quantity of fluid was reduced to a drop, the galvanometer gave no indication.

24. Attempts to obtain similar effects to these by the use of wires conveying ordinary electricity were doubtful in the results. A compound helix similar to that already described (6.), and containing eight elementary helices was used. Four of the helices had their similar ends bound together by wire, and the two general terminations thus produced connected with the small magnetising helix contained an unmagnetised needle (13.). The other four helices were similarly arranged, but their ends connected with a Leyden jar. On passing the discharge, the needle was found to be a magnet; but it appeared probable that a part of the electricity of the jar had passed off to the small helix, and so magnetised the needle. There was indeed no reason to expect that the electricity of a jar possessing as it does great tension, would not diffuse itself through all the metallic matter interposed between the coatings.

25. Still it does not follow that the discharge of ordinary electricity through a wire does not produce analogous phenomena to those arising from voltaic electricity; but as it appears impossible to separate the effects produced at the moment when the discharge begins to pass, from the equal and contrary effects produced when it ceases to pass (16.), inasmuch as with ordinary electricity these periods are simultaneous, so there can be scarcely any hope that in this form of the experiment they can be perceived.

26. Hence it is evident that currents of voltaic electricity present phenomena of induction somewhat analogous to those produced by electricity of tension, although, as will be seen hereafter, many differences exist between them. The result is the production of other currents, (but which are only momentary,) parallel, or tending to parallelism, with the inducing current. By reference to the poles of the needle formed in the indicating helix (13.14.) and to the deflections of the galvanometer-needle (11.), it was found in all cases that the induced current, produced by the first action of the inducing current, was in the contrary direction to the latter, but that the current produced by the cessation of the inducing current was in the same direction. For the purpose of avoiding periphrasis, I propose to call this action of the current 



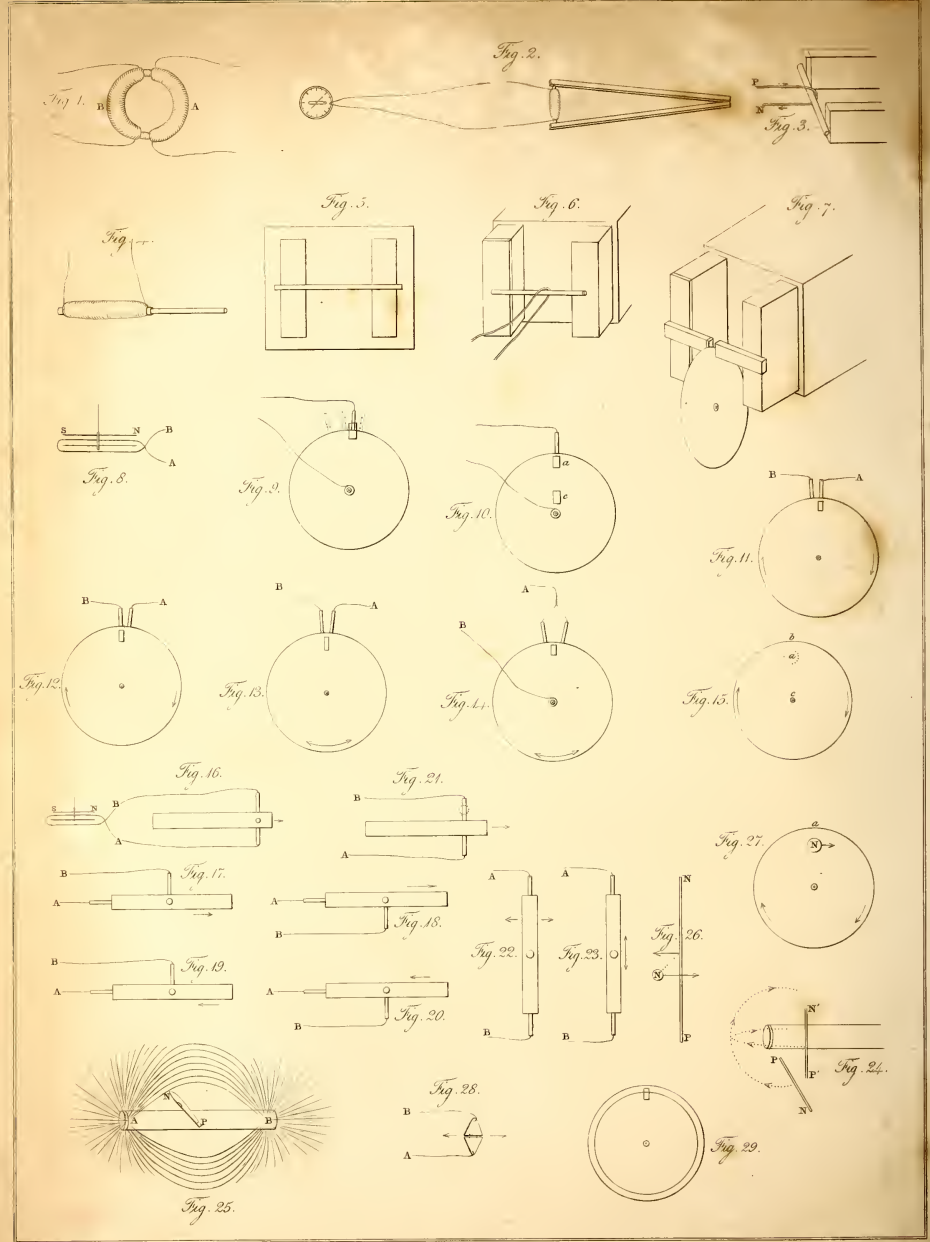


from the voltaic battery, volta-electric induction. The properties of the wire, after induction has developed the first current, and whilst the electricity from the battery continues to flow through its inducing neighbour (10.18.), constitute a peculiar electric condition, the consideration of which will be resumed hereafter. All these results have been obtained with a voltaic apparatus consisting of a single pair of plates.

\section{\$2. Evolution of Electricity from Magnetism.}

27. A welded ring was made of soft round bar-iron, the metal being seven eighths of an inch in thickness, and the ring six inches in external diameter. Three helices were put round one part of this ring, each containing about twenty-four feet of copper wire one twentieth of an inch thick ; they were insulated from the iron and each other, and superposed in the manner before described (6.), occupying about nine inches in length upon the ring. They could be used separately or arranged together; the group may be distinguished by the mark A (Pl. III. fig. 1.). On the other part of the ring about sixty feet of similar copper wire in two pieces were applied in the same manner, forming a helix B, which had the same common direction with the helices of A, but being separated from it at each extremity by about half an inch of the uncovered iron.

28. The helix B was connected by copper wires with a galvanometer three feet from the ring. The wires of $\mathrm{A}$ were connected end to end so as to form one long helix, the extremities of which were connected with a battery of ten pairs of plates four inches square. The galvanometer was immediately affected, and to a degree far beyond what has been described, when with a battery of tenfold power helices without iron were used (10.); but though the contact was continued, the effect was not permanent, for the needle soon came to rest in its natural position, as if quite indifferent to the attached electro-magnetic arrangement. Upon breaking the contact with the battery, the needle was again powerfully deflected, but in the contrary direction to that induced in the first instance.

29. Upon arranging the apparatus so that $B$ should be out of use, the galvanometer be connected with one of the three wires of $\mathrm{A}$, and the other two made into a helix through which the current from the trough (28.) was passed; similar but rather more powerful effects were produced. 


\section{MR. FARADAY'S EXPERIMENTAL RESEARCHES IN ELECTRICITY.}

30. When the battery contact was made in one direction, the galvanometer needle was deflected on the one side; if made in the other direction, the deflection was on the other side. The deflection on breaking the battery contact was always the reverse of that produced by completing it. The deflection on making a battery contact always indicated an induced current in the opposite direction to that from the battery ; but on breaking the contact the deflection indicated an induced current in the same direction as that of the battery. No making or breaking of the contact at B side, or in any part of the galvanometer circuit, produced any effect at the galvanometer. No continuance of the battery current caused any deflection of the galvanometer-needle. As the above results are common to all these experiments, and to similar ones with ordinary magnets to be hereafter detailed, they need not be again particularly described.

31. Upon using the power of one hundred pair of plates (10.) with this ring, the impulse at the galvanometer, when contact was completed or broken, was so great as to make the needle spin round rapidly four or five times before the air and terrestrial magnetism could reduce its motion to mere oscillation.

32. By using charcoal at the ends of the $B$ helix, a minute spark could be perceived when the contact of the battery with $A$ was completed. This spark could not be due to any diversion of a part of the current of the battery through the iron to the helix B ; for when the battery contact was continued, the galvanometer still resumed its perfectly indifferent state (28.). The spark was rarely seen on breaking contact. A small platina wire could not be ignited by this induced current; but there seems every reason to believe that the effect would be obtained by using a stronger original current or a more powerful arrangement of helices.

33. A feeble voltaic current was sent through the helix B and the galvanometer, so as to deflect the needle of the latter $30^{\circ}$ or $40^{\circ}$, and then the battery of one hundred pairs of plates connected with $\mathrm{A}$; but after the first effect was over, the galvanometer needle resumed exactly the position due to the feeble current transmitted by its own wire. This took place in whichever way the battery contacts were made, and shows that here again (20.) no permanent influence of the currents upon each other, as to their quantity and tension, exists.

34. Another arrangement was then used connecting the former experiments on volta-electric induction with the present. A combination of helices like 
that already described (6.) was constructed upon a hollow cylinder of pasteboard : there were eight lengths of copper wire, containing altogether 220 feet ; four of these helices were connected end to end, and then with the galvanometer (7.); the other intervening four were also connected end to end, and the battery of one hundred pairs discharged through them. In this form the effect on the galvanometer was hardly sensible (11.), but magnets could be made by the induced current (13.). But when a soft iron cylinder seven eighths of an inch thick, and twelve inches long, was introduced into the pasteboard tube, surrounded by the helices, then the induced current affected the galvanometer powerfully, and with all the phenomena just described (30.). It possessed also the power of making magnets with more energy, apparently, than when no iron cylinder was present.

35. When the iron cylinder was replaced by an equal cylinder of copper, no effect beyond that of the helices alone was produced. The iron cylinder arrangement was not so powerful as the ring arrangement already described (27.).

36. Similar effects were then produced by ordinary magnets : thus the hollow helix just described (34.) had all its elementary helices connected with the galvanometer by two copper wires, each five feet in length; the soft iron cylinder was introduced into its axis ; a couple of bar magnets, each twenty-four inches long, were arranged with their opposite poles at one end in contact, so as to resemble a horse-shoe magnet, and then contact made between the other poles and the ends of the iron cylinder, so as to convert it for the time into a magnet (fig. 2.) : by breaking the magnetic contacts, or reversing them, the magnetism of the iron cylinder could be destroyed or reversed at pleasure.

37. Upon making magnetic contact, the needle was deflected; continuing the contact, the needle became indifferent, and resumed its first position; on breaking the contact, it was again deflected, but in the opposite direction to the first effect, and then it again became indifferent. When the magnetic contacts were reversed, the deflections were reversed.

38. When the magnetic contact was made, the deflection was such as to indicate an induced current of electricity in the opposite direction to that fitted to form a magnet having the same polarity as that really produced by contact with the bar magnets. Thus when the marked and unmarked poles were placed as in fig. 3 , the current in the helix was in the direction represented, $P$ being sup- 
posed to be the end of the wire going to the positive pole of the battery, or that end towards which the zinc plates face, and $\mathbf{N}$ the negative wire. Such a current would have converted the cylinder into a magnet of the opposite kind to that formed by contact with the poles A and B; and such a current moves in the opposite direction to the currents which in M. AmpEre's beautiful theory are considered as constituting a magnet in the position figured *.

39. But as it might be supposed that in all the preceding experiments of this section it was by some peculiar effect taking place during the formation of the magnet, and not by its mere virtual approximation, that the momentary induced current was excited, the following experiment was made. All the similar ends of the compound hollow helix (34.) were bound together by copper wire, forming two general terminations, and these were connected with the galvanometer. The soft iron cylinder (34.) was removed, and a cylindrical magnet, three quarters of an inch in diameter and eight inches and a half in length, used instead. One end of this magnet was introduced into the axis of the helix (fig. 4.), and then, the galvanometer-needle being stationary, the magnet was suddenly thrust in ; immediately the needle was deflected in the same direction as if the magnet had been formed by either of the two preceding processes (34. 36.). Being left in, the needle resumed its first position, and then the magnet being withdrawn the needle was deflected in the opposite direction. These effects were not great; but by introducing and withdrawing the magnet, so that the impulse each time should be added to those previously communicated to the needle, the latter could be made to vibrate through an arc of $180^{\circ}$ or more.

40. In this experiment the magnet must not be passed entirely through the

* The relative position of an electric current and a magnet is by most persons found very difficult to remember, and three or four helps to the memory have been devised by M. AMPERE and others. I venture to suggest the following as a very simple and effectual assistance in these and similar latitudes. Let the experimenter think he is looking down upon a dipping needle, or upon the pole of the earth, and then let him think upon the direction of the motion of the hands of a watch, or of a screw moving direct; currents in that direction round a needle would make it into such a magnet as the dipping needle, or would themselves constitute an electro-magnet of similar qualities; or if brought near a magnet would tend to make it take that direction; or would themselves be moved into that position by a magnet so placed; or in M. Amperr's theory are considered as moving in that direction in the magnet. These two points of the position of the dipping-needle and the motion of the watch-hands being remembered, any other relation of the current and magnet can be at once deduced from it. 
helix, for then a second action occurs. When the magnet is introduced, the needle at the galvanometer is deflected in a certain direction; but being in, whether it be pushed quite through or withdrawn, the needle is deflected in a direction the reverse of that previously produced. When the magnet is passed in and through at one continuous motion, the needle moves one way, is then suddenly stopped, and finally moves the other way.

41. If such a hollow helix as that described (34.) be laid east and west (or in any other constant position), and a magnet be retained east and west, its marked pole always being one way; then whichever end of the helix the magnet goes in at, and consequently whichever pole of the magnet enters first, still the needle is deflected the same way: on the other hand, whichever direction is followed in withdrawing the magnet, the deflection is constant, but contrary to that due to its entrance.

42. These effects are simple consequences of the law hereafter to be described (114).

43. When the eight elementary helices were made one long helix, the effect was not so great as in the arrangement described. When only one of the eight helices was used, the effect was also much diminished. All care was taken to guard against any direct action of the inducing magnet upon the galvanometer, and it was found that by moving the magnet in the same direction, and to the same degree on the outside of the helix, no effect on the needle was produced.

44. The Royal Society are in possession of a large compound magnet formerly belonging to Dr. Gowin Knight, which, by permission of the President and Council, I was allowed to use in the prosecution of these experiments : it is at present in the charge of Mr. Christie, at his house at Woolwich, where, by Mr. ChristiE's kindness, I was at liberty to work; and I have to acknowledge my obligations to him for his assistance in all the experiments and observations made with it. This magnet is composed of about 450 bar magnets, each fifteen inches long, one inch wide, and half an inch thick, arranged in a box so as to present at one of its extremities two external poles (fig. 5.). These poles projected horizontally six inches from the box, were each twelve inches high and three inches wide. They were nine inches apart ; and when a soft iron cylinder, three quarters of an inch in diameter and twelve inches long, was put across 
from one to the other, it required a force of nearly one hundred pounds to break the contact. The pole to the left in the figure is the marked pole *.

45. The indicating galvanometer, in all experiments made with this magnet, was about eight feet from it, not directly in front of the poles, but about $16^{\circ}$ or $17^{\circ}$ on one side. It was found that on making or breaking the connexion of the poles by soft iron, the instrument was slightly affected ; but all error of observation arising from this cause was easily and carefully avoided.

46. The electrical effects exhibited by this magnet were very striking. When a soft iron cylinder thirteen inches long was put through the compound hollow helix, with its ends arranged as two general terminations (39.), these connected with the galvanometer, and the iron cylinder brought in contact with the two poles of the magnet (fig. 5.), so powerful a rush of electricity took place that the needle whirled round many times in succession $\uparrow$.

47. Notwithstanding this great power, if the contact was continued, the needle resumed its natural position, being entirely uninfluenced by the position of the helix (30.). But on breaking the magnetic contact, the needle was whirled round in the opposite direction with a force equal to the former.

48. A piece of copper plate wrapped once round the iron cylinder like a socket, but with interposed paper to prevent contact, had its edges connected with the wires of the galvanometer. When the iron was brought in contact with the poles, the galvanometer was strongly affected.

49. Dismissing the helices and sockets, the galvanometer wire was passed over, and consequently only half round the iron cylinder (fig. 6.); but even then a strong effect upon the needle was exhibited, when the magnetic contact was made or broken.

50. As the helix with its iron cylinder was brought towards the magnetic poles, but without making contact, still powerful effects were produced. When the helix, without the iron cylinder, and consequently containing no metal but

* To avoid any confusion as to the poles of the magnet, I shall designate the pole pointing to the north as the marked pole; I may occasionally speak of the north and south ends of the needle, but do not mean thereby north and south poles. That is by many considered the true north pole of a needle which points to the south; but in this country it is often called the south pole.

+ A soft iron bar in the form of a lifter to a horse-shoe magnet, when supplied with a coil of this kind round the middle of it, becomes, by juxta-position with a magnet, a ready source of a brief but determinate current of electricity. 
copper, was approached to, or placed between the poles (44.), the needle was thrown $80^{\circ}, 90^{\circ}$, or more, from its natural position. The inductive force was of course greater, the nearer the helix, either with or without its iron cylinder, was brought to the poles; but otherwise the same effects were produced, whether the helix, \&c. was or was not brought into contact with the magnet; i. e. no permanent effect on the galvanometer was produced; and the effects of approximation and removal were the reverse of each other (30.).

51. When a bolt of copper corresponding to the iron cylinder was introduced, no greater effect was produced by the helix than without it. But when a thick iron wire was substituted, the magneto-electric induction was rendered sensibly greater.

52. The direction of the electric current produced in all these experiments with the helix, was the same as that already described (38.) as obtained with the weaker bar magnets.

53. A spiral containing fourteen feet of copper wire, being connected with the galvanometer, and approximated directly towards the marked pole in the line of its axis, affected the instrument strongly; the current induced in it was in the reverse direction to the current theoretically considered by M. AMPERE as existing in the magnet (38.), or as the current in an electro-magnet of similar polarity. As the spiral was withdrawn, the induced current was reversed.

54. A similar spiral had the current of eighty pairs of 4-inch plates sent through it so as to form an electro-magnet, and then the other spiral connected with the galvanometer (53.) approximated to it ; the needle vibrated, indicating a current in the galvanometer spiral the reverse of that in the battery spiral (18. 26.). On withdrawing the latter spiral, the needle passed in the opposite direction.

55. Single wires, approximated in certain directions towards the magnetic pole, had currents induced in them. On their removal, the currents were inverted. In such experiments the wires should not be removed in directions different to those in which they were approximated; for then occasionally complicated and irregular effects are produced, the causes of which will be very evident in the fourth part of this paper.

56. All attempts to obtain chemical effects by the induced current of elecMDCCCXXXII. 
tricity failed, though the precautions before described (22.), and all others that could be thought of, were employed. Neither was any sensation on the tongue, or any convulsive effect upon the limbs of a frog, produced. Nor could charcoal or fine wire be ignited (133.). But upon repeating the experiments more at leisure at the Royal Institution, with an armed loadstone belonging to Professor Danielu and capable of lifting about thirty pounds, a frog was very powerfully convulsed each time magnetic contact was made. At first the convulsions could not be obtained on breaking magnetic contact; but conceiving the deficiency of effect was because of the comparative slowness of separation, the latter act was effected by a blow, and then the frog was convulsed strongly. The more instantaneous the union or disunion is effected, the more powerful the convulsion. I thought also I could perceive the sensation upon the tongue and the flash before the eyes; but I could obtain no evidence of chemical decomposition.

-57. The various experiments of this section prove, I think, most completely the production of electricity from ordinary magnetism. That its intensity should be very feeble and quantity small, cannot be considered wonderful, when it is remembered that like thermo-electricity it is evolved entirely within the substance of metals retaining all their conducting power. But an agent which is conducted along metallic wires in the manner described; which, whilst so passing possesses the peculiar magnetic actions and force of a current of electricity; which can agitate and convulse the limbs of a frog; and which, finally, can produce a spark by its discharge through charcoal (32.), can only be electricity. As all the effects can be produced by ferruginous electro-magnets (34.), there is no doubt that arrangements like the magnets of Professors Moll, Henry, Ten Eyke, and others, in which as many as two thousand pounds have been lifted, may be used for these experiments; in which case not only a brighter spark may be obtained, but wires also ignited, and, as the current can pass liquids (23.), chemical action be produced. These effects are still more likely to be obtained when the magneto-electric ariangements to be explained in the fourth section are excited by the powers of such apparatus.

58. The similarity of action, almost amounting to identity, between common magnets and either electro-magnets or volta-electric currents, is strikingly in accordance with and confirmatory of M. AMPERE's theory, and furnishes power- 
ful reasons for believing that the action is the same in both cases; but, as a distinction in language is still necessary, I propose to call the agency thus exerted by ordinary magnets, magneto-electric or magnelectric induction (26.).

59. The only difference which powerfully strikes the attention as existing between volta-electric and magneto-electric induction, is the suddenness of the former and the sensible time required by the latter; but even in this early state of investigation there are circumstances which seem to indicate, that upon further inquiry this difference will, as a philosophical distinction, disappear (68.).

\section{§. 3. New Electrical State or Condition of Matter*.}

60. Whilst the wire is subject to either volta-electric or magneto-electric induction, it appears to be in a peculiar state ; for it resists the formation of an electrical current in it, whereas, if in its common condition, such a current would be produced; and when left uninfluenced it has the power of originating a current, a power which the wire does not possess under common circumstances. This electrical condition of matter has not hitherto been recognised, but it probably exerts a very important influence in many if not most of the phenomena produced by currents of electricity. For reasons which will immediately appear (71.), I have, after advising with several learned friends, ventured to designate it as the electro-tonic state.

61. This peculiar condition shows no known electrical effects whilst it continues; nor have I yet been able to discover any peculiar powers cxerted, or properties possessed, by matter whilst retained in this state.

62. It shows no reaction by attractive or repulsive powers. The various experiments which have been made with powerful magnets upon such metals as copper, silver, and generally those substances not magnetic, prove this point ; for the substances experimented upon, if electrical conductors, must have acquired this state; and yet no evidence of attractive or repulsive powers has been observed. I have placed copper and silver discs, very delicately sus-

* This section having been read at the Royal Society and reported upon, and having also, in consequence of a letter from myself to M. HAcheTre, been noticed at the French Institute, I feel bound to let it stand as part of the paper; but later investigations (intimated 73.76.77.) of the laws governing these phenomena, induce me to think that the latter can be fully explained without admitting the electro-tonic state. My views on this point will appear in the second series of these researches.-M. F. 
pended on torsion balances in vacuo, near to the poles of very powerful magnets, yet have not been able to observe the least attractive or repulsive force.

63. I have also arianged a fine slip of gold-leaf very near to a bar of copper, the two being in metallic contact by mercury at their extremities. These have been placed in vacuo, so that metal rods connected with the extremities of the arrangement should pass through the sides of the vessel into the air. I have then moved powerful magnetic poles, by this arrangement, in various directions, the metallic circuit on the outside being sometimes completed by wires, and sometimes broken. But I never could obtain any sensible motion of the goldleaf, either directed to the magnet or towards the collateral bar of copper, which must have been, as far as induction was concerned, in a similar state to itself.

64. In some cases it has been supposed that, under such circumstances, attractive and repulsive forces have been exhibited, i. e. that such bodies have become slightly magnetic. But the phenomena now described, in conjunction with the confidence we may reasonably repose in M. AMpere's theory of magnetism, tend to throw doubt on such cases; for if magnetism depend upon the attraction of electrical currents, and if the powerful currents at first excited, both by volta-electric and magneto-electric induction, instantly and naturally cease (12.28.47.), causing at the same time an entire cessation of magnetic effects at the galvanometer needle, then there can be little or no expectation that any substances not partaking of the peculiar relation in which iron, nickel, and one or two other bodies, stand, should exhibit magnetoattractive powers. It seems far more probable, that the extremely feeble permanent effects observed have been due to traces of iron, or some other unrecognised cause not magnetic.

65. This peculiar condition exerts no retarding or accelerating power upon electrical currents passing through metal thus circumstanced (20.33.). Neither could any such power upon the inducing current itself be detected; for when masses of metal, wires, helices, \&c. were arranged in all possible ways by the side of a wire or helix, carrying a current measured by the galvanometer (20.), not the slightest permanent change in the indication of the instrument could be perceived. Metal in the supposed peculiar state, therefore, conducts electricity in all directions with its ordinary facility, or, in other words, its conducting power is not sensibly altered by it. 
66. All metals take on the peculiar state. This is proved in the preceding experiments with copper and iron (9.), and with gold, silver, tin, lead, zinc, antimony, bismuth, mercury, \&c. by experiments to be described in the fourth part (132.), admitting of easy application. With regard to iron, the experiments prove the thorough and remarkable independence of these phenomena of induction, and the ordinary magnetical appearances of that metal.

67. This state is altogether the effect of the induction exerted, and ceases as soon as the inductive force is removed. It is the same state, whether produced by the collateral passage of voltaic currents (26.), or the formation of a magnet (34. 36.), or the mere approximation of a magnet (39.50.); and is a strong proof in addition to those advanced by M. AMpEre, of the identity of the agents concerned in these several operations. It probably occurs, momentarily, during the passage of the common electric spark (24.), and may perhaps be obtained hereafter in bad conductors by weak electrical currents or other means (74. 76.).

68. The state appears to be instantly assumed (12.), requiring hardly a sensible portion of time for that purpose. The difference of time between volta-electric and magneto-electric induction, rendered evident by the galvanometer (59.), may probably be thus explained. When a voltaic current is sent through one of two parallel wires, as those of the hollow helix (34.), a current is produced in the other wire, as brief in its continuance as the time required for a single action of this kind, and which, by experiment, is found to be inappreciably small. The action will seem still more instantaneous, because, as there is an accumulation of power in the poles of the battery before contact, the first rush of electricity in the wire of communication is greater than that sustained after the contact is completed; the wire of induction becomes at the moment electro-tonic to an equivalent degree, which the moment after sinks to the state in which the continuous current can sustain it, but in sinking causes an opposite induced current to that at first produced. The consequence is, that the first induced wave of electricity more resembles that from the discharge of an electric jar, than it otherwise would do.

69. But when the iron cylinder is put into the same helix (34.), previous to the connexion being made with the battery, then the current from the latter may be considered as active in inducing innumerable currents of a sinilar kind 
to itself in the iron, rendering it a magnet. This is known by experiment to occupy time; for a magnet so formed, even of soft iron, does not rise to its fullest intensity in an instant, and it may be because the currents within the iron are successive in their formation or arrangement. But as the magnet can induce, as well as the battery current, the combined action of the two continues to evolve induced electricity, until their joint effect is at a maximum, and thus the existence of the deflecting force is prolonged sufficiently to overcome the inertia of the galvanometer needle.

70. In all those cases where the helices or wires are advanced towards or taken from the magnet (50.55.), the direct or inverted current of induced electricity continues for the time occupied in the advance or recession; for the electro-tonic state is rising to a higher or falling to a lower degree during that time, and the change is accompanied by its corresponding evolution of electricity; but these form no objections to the opinion that the electro-tonic state is instantly assumed.

71. This peculiar state appears to be a state of tension, and may be considered as equivalent to a current of electricity, at least equal to that produced either when the condition is induced or left at liberty. The current evolved, however, first or last, is not to be considered a measure of the degree of tension to which the electro-tonic state has risen; for as the metal retains its conducting powers unimpaired (65.), and as the electricity evolved is but for a moment, (the peculiar state being instantly assumed and lost (68.),) the electricity which may be led away by long wire conductors, offering obstruction in their substance proportionate to their small lateral and extensive linear dimensions, can be but a very small portion of that really evolved within the mass at the moment it assumes this condition. Insulated helices and portions of metal instantly assumed the state; and no traces of electricity could be discovered in them, however quickly the contact with the electrometer was made, after they were put under induction, either by the current from the battery or the magnet. A single drop of water or a small piece of moistened paper (23. 56.) was obstacle sufficient to stop the current through the conductors, the electricity evolved returning to a state of equilibrium through the metal itself, and consequently in an unobserved manner.

72. The tension of this state may therefore be comparatively very great. 
But whether great or small, it is hardly conceivable that it should exist without exerting a reaction upon the original inducing current, and producing equilibrium of some kind. It might be anticipated that this would give rise to a retardation of the original current; but $I$ have not been able to ascertain that this is the case. Neither have $I$ in any other way as yet been able to distinguish effects attributable to such a reaction.

73. All the results favour the notion that the electro-tonic state relates to the particles, and not to the mass, of the wire or substance under induction, being in that respect different to the induction exerted by electricity of tension. If so, the state may be assumed in liquids when no electrical current is sensible, and even in non-conductors; the current itself, when it occurs, being as it were a contingency due to the existence of conducting power, and the momentary propulsive force exerted by the particles during their arrangement. Even when conducting power is equal, the currents of electricity, which as yet are the only indicators of this state, may be unequal, because of differences as to number, size, electrical condition, \&c. \&c. in the particles themselves. It will only be after the laws which govern this new state are ascertained, that we shall be able to predict what is the true condition of, and what are the electrical results obtainable from, any particular substance.

74. The current of electricity which induces the electro-tonic state in a neighbouring wire, probably induces that state also in its own wire; for when by a current in one wire a collateral wire is made electro-tonic, the latter state is not rendered any way incompatible or interfering with a current of electricity passing through it (62.). If, therefore, the current were sent through the second wire instead of the first, it does not seem probable that its inducing action upon the second would be less, but on the contrary more, because the distance between the agent and the matter acted upon would be very greatly diminished. A copper bolt had its extremities connected with a galvanometer, and then the poles of a battery of one hundred pairs of plates connected with the bolt, so as to send the current through it; the voltaic circuit was then suddenly broken, and the galvanometer observed for any indications of a return current through the copper bolt due to the discharge of its supposed electro-tonic state. No effect of the kind was obtained, nor indeed, for two reasons, ought it to be expected; for first, as the cessation of 


\section{4}

MR. FARADAY'S EXPERIMENTAL RESEARCHES IN ELECTRICITY.

induction and the discharge of the electro-tonic condition are simultaneous, and not successive, the return current would only be equivalent to the neutralization of the last portion of the inducing current, and would not therefore show any alteration of direction; or assuming that time did intervene, and that the latter current was really distinct from the former, its short, sudden character (12. 26.) would prevent it from being thus recognised.

75 . No difficulty arises, I think, in considering the wire thus rendered electrotonic by its own current more than by any external current, especially when the apparent non-interference of that state with currents is considered (62.71.). The simultaneous existence of the conducting and electro-tonic states finds an analogy in the manner in which electrical currents can be passed through magnets where it is found that both the currents passed, and those of the magnets, preserve all their properties distinct from each other, and exert their mutual actions.

76. The reason given with regard to metals extends also to fluids and all other conductors, and leads to the conclusion that when electric currents are passed through them they also assume the electro-tonic state. Should that prove to be the case, its influence in voltaic decomposition, and the transference of the elements to the poles, can hardly be doubted. In the electro-tonic state the homogeneous particles of matter appear to have assumed a regular but forced electrical arrangement in the direction of the current, which if the matter be undecomposable produces, when relieved, a return current; but in decomposable matter this forced state may be sufficient to make an elementary particle leave its companion, with which it is in a constrained condition, and associate with the neighbouring similar particle, in relation to which it is in a more natural condition, the forced electrical arrangement being itself discharged or relieved, at the same time, as effectually as if it had been freed from induction. But as the original voltaic current is continued, the electro-tonic state may be instantly renewed, producing the forced arrangement of the compound particles, to be as instantly discharged by a transference of the elementary particles of the opposite kind in opposite directions, but parallel to the current. Even the differences between common and voltaic electricity when applied to effect chemical decomposition, which Dr. WoLlaston has pointed out *,

* Philosophical Transactions, 1801. p. 247. 
seem explicable by the circumstances connected with the induction of electricity from these two sources (25.). But as I have reserved this branch of the inquiry, that I might follow out the investigations contained in the present paper, I refrain (though much tempted) from offering further speculations.

77. Marianini has discovered and described a peculiar affection of the surfaces of metallic discs, when, being in contact with humid conductors, a current of electricity is passed through them ; they are then capable of producing a reverse current of electricity, and Marianini has well applied the effect in explanation of the phenomena of Ritter's piles*. M. A. DE LA Rive has described a peculiar property acquired by metallic conductors, when being immersed in a liquid as poles, they have completed, for some time, the voltaic circuit, in consequence of which, when separated from the battery and plunged in the same fluid, they themselves produce an electric current $\uparrow$. M. A. VAN BEEK has detailed cases in which the electrical relation of one metal in contact with another has been preserved after separation, and accompanied by its corresponding chemical effects $*$. These states and results appear to differ from the electro-tonic state and its phenomena; but the true relation of the former to the latter can only be decided when our knowledge of all these phenomena has been enlarged.

78. I had occasion in the commencement of this paper (2.) to refer to an experiment by Ampere, as one of those dependent upon the electrical induction of currents made prior to the present investigation, and have arrived at conclusions which seem to imply doubts of the accuracy of the experiment (62, \&c.) : it is therefore due to M. Ampere that I should attend to it more distinctly. When a disc of copper (says M. Ampere) was suspended by a silk thread and surrounded by a belix or spiral, and when the charge of a powerful voltaic battery was sent through the spiral, a strong magnet at the same time being presented to the copper disc, the latter turned at the moment to take a position of equilibrium, exactly as the spiral itself would have turned had it been free to move. I have not been able to obtain this effect, nor indeed any motion; but the cause of my failure in the latter point may be due to the momentary existence of the current not allowing time for the inertia of the plate to be overcome (11. 12.). M. Ampere has perhaps succeeded in obtaining motion

* Annales de Chimie, XXXVIII. 5. MDCCCXXXII.
+ Ibid. XXVIII. 190.
† Ibid. XXXVIII. 49. 
from the superior delicacy and power of his electro-magnetical apparatus, or he may have obtained only the motion due to cessation of action. But all my results tend to invert the sense of the proposition stated by M. Ampere, "that a current of electricity tends to put the electricity of conductors near which it passes in motion in the same direction," for they indicate an opposite direction for the produced current (26. 53.); and they show that the effect is momentary, and that it is also produced by magnetic induction, and that certain other extraordinary effects follow thereupon.

79. The momentary existence of the phenomena of induction now described is sufficient to furnish abundant reasons for the uncertainty or failure of the experiments hitherto made to obtain electricity from magnets, or to effect chemical decomposition or arrangement by their means*.

80. It also appears capable of explaining fully the remarkable phenomena observed by M. Arago between metals and magnets when either are moving (120.), as well as most of the results obtained by Sir John Herschel, Messrs. Babbage, HaRris, and others, in repeating his experiments; accounting at the same time perfectly for what at first appeared inexplicable; namely, the nonaction of the same metals and magnets when at rest. These results, which also afford the readiest means of obtaining electricity from magnetism, I shall now proceed to describe.

\section{\$4. Explication of Arago's Magnetic Phenomena.}

81. If a plate of copper be revolved close to a magnetic needle, or magnet,

* The Lycée, No. 36, for January 1st, has a long and rather premature article, in which it endeavours to show anticipations by French philosophers of my researches. It however mistakes the erroneous results of MM. FresNeL and AMPERE for true ones, and then imagines my true results are like those erroneous ones. I notice it here, however, for the purpose of doing honour to FRESNRL in a much higher degree than would have been merited by a feeble anticipation of the present investigations. That great philosopher, at the same time with myself and fifty other persons, made experiments which the present paper proves could give no expected result. He was deceived for the moment, and published his imaginary success; but on more carefully repeating his trials, he could find no proof of their accuracy; and, in the high and pure philosophic desire to remove error as well as discover truth, he recanted his first statement. The example of BenzeLIUs regarding the first Thorina is another instance of this fine feeling; and as occasions are not rare, it would be to the dignity of science if such examples were more frequently followed.-February 10th, 1832. 
suspended in such a way that the latter may rotate in a plane parallel to that of the former, the magnet tends to follow the motion of the plate; or if the magnet be revolved, the plate tends to follow its motion; and the effect is so powerful, that magnets or plates of many pounds weight may be thus carried round. If the magnet and plate be at rest relative to each other, not the slightest effect, attractive or repulsive, or of any kind, can be observed between them (62.). This is the phenomenon discovered by M. Arago; and he states that the effect takes place not only with all metals, but with solids, liquids, and even gases, i. e. with all substances (130.).

82. Mr. Babbage and Sir John Herschel, on conjointly repeating the experiments in this country*, could obtain the effects only with the metals, and with carbon in a peculiar state (from gas retorts), i. e. only with excellent conductors of electricity. They refer the effect to magnetism induced in the plate by the magnet; the pole of the latter causing an opposite pole in the nearest part of the plate, and round this a more diffuse polarity of its own kind (120.). The essential circumstance in producing the rotation of the suspended magnet is, that the substance revolving below it shall acquire and lose its magnetism in a finite time, and not instantly (124.). This theory refers the effect to an attractive force, and is not agreed to by the discoverer, M. Arago, nor by M. Ampere, who quote against it the absence of all attraction when the magnet and metal are at rest (62. 126.), although the induced magnetism should still remain; and who, from experiments made with a long dipping needle, conceive the action to be always repulsive (125.).

83. Upon obtaining electricity from magnets by the means already described (36. 46.), I hoped to make the experiment of M. AraGo a new source of electricity; and did not despair, by reference to terrestrial magneto-electric induction, of being able to construct a new electrical machine. Thus stimulated, numerous experiments were made with the magnet of the Royal Society at Mr. Christie's house, in all of which I had the advantage of his assistance. As many of these were in the course of the investigation superseded by more perfect arrangements, I shall consider myself at liberty to rearrange them in a manner calculated to convey most readily what appears to me to be a correct view of the nature of the phenomena.

\footnotetext{
* Philosophical Transactions, 1825. p. 467.
} 
84. The magnet has been already described (44.). To concentrate the poles, and bring them nearer to each other, two iron or steel bars, each about six or seven inches long, one inch wide, and half an inch thick, were put across the poles as in fig. 7, and being supported by twine from slipping, could be placed as near to or far from each other as was required. Occasionally two bars of soft iron were employed, so bent that when applied, one to each pole, the two smaller resulting poles were vertically over each other, either being uppermost at pleasure.

85. A disc of copper, twelve inches in diameter, and about one fifth of an inch in thickness, fixed upon a brass axis, was mounted in frames so as to be revolved either vertically or horizontally, its edge being at the same time introduced more or less between the magnetic poles (fig. 7.). The edge of the plate was well amalgamated for the purpose of obtaining a good but moveable contact ; a part round the axis was also prepared in a similar manner.

86. Conductors or collectors of copper and lead were constructed so as to come in contact with the edge of the copper disc (85.), or with other forms of plates hereafter to be described (101.). These conductors were about four inches long, one third of an inch wide, and one fifth of an inch thick; one end of each was slightly grooved, to allow of more exact adaptation to the somewhat convex edge of the plates, and then amalgamated. Copper wires, one sixteenth of an inch in thickness, attached, in the ordinary manner, by convolutions to the other ends of these conductors, passed away to the galvanometer.

87. The galvanometer was roughly made, yet sufficiently delicate in its indications. The wire was of copper covered with silk, and made sixteen or eighteen convolutions. Two sewing-needles were magnetized and passed through a stem of dried grass parallel to each other, but in opposite directions, and about half an inch apart; this system was suspended by a fibre of unspun silk, so that the lower needle should be between the convolutions of the multiplier, and the upper above them. The latter was by much the most powerful magnet, and gave terrestrial direction to the whole; fig. 8. represents the direction of the wire and of the needles when the instrument was placed in the magnetic meridian; the ends of the wires are marked A and B for convenient reference hereafter. The letters $\mathrm{S}$ and $\mathrm{N}$ designate the south and north 
ends of the needle when affected merely by terrestrial magnetism; the end $\mathrm{N}$ is therefore the marked pole (44.). The whole instrument was protected by a glass jar, and stood, as to position and distance relative to the large magnet, under the same circumstances as before (45.).

88. All these arrangements being made, the copper disc was adjusted as in fig. 7, the small magnetic poles being about half an inch apart, and the edge of the plate inserted about half their width between them. One of the galvanometer wires was passed twice or thrice loosely round the brass axis of the plate, and the other attached to a conductor (86.), which itself was retained by the hand in contact with the amalgamated edge of the disc at the part immediately between the magnetic poles. Under these circumstances all was quiescent, and the galvanometer exhibited no effect. But the instant the plate moved, the galvanometer was influenced, and by revolving the plate quickly the needle could be deflected $90^{\circ}$ or more.

89. It was difficult under the circumstances to make the contact between the conductor and the edge of the revolving disc uniformly good and extensive; it was also difficult in the first experiments to obtain a regular velocity of rotation: both these causes tended to retain the needle. in a continual state of vibration; but no difficulty existed in ascertaining to which side it was deflected, or generally, about what line it vibrated. Afterwards, when the experiments were made more carefully, a permanent deflection of the needle of nearly $45^{\circ}$ could be sustained.

90. Here therefore was demonstrated the production of a permanent current of electricity by ordinary magnets (57.).

91. When the motion of the disc was reversed, every other circumstance remaining the same, the galvanometer needle was deflected with equal power as before; but the deflection was on the opposite side, and the current of electricity evolved, therefore, the reverse of the former.

92. When the conductor was placed on the edge of the disc a little to the right or left, as in the dotted positions fig. 9, the current of electricity was still evolved, and in the same direction as at first (88. 91.). This occurred to a considerable distance, i. e. $50^{\circ}$ or $60^{\circ}$ on each side of the place of the magnetic poles. The current gathered by the conductor and conveyed to the galvanometer was of the same kind on both sides of the place of greatest inten- 
sity, but gradually diminished in force from that place. It appeared to be equally powerful at equal distances from the place of the magnetic poles, not being affected in that respect by the direction of the rotation. When the rotation of the disc was reversed, the direction of the current of electricity was reversed also; but the other circumstances were not affected.

93. On raising the plate, so that the magnetic poles were entirely hidden from each other by its intervention, ( $a$. fig. 10,) the same effects were produced in the same order, and with equal intensity as before. On raising it still higher, so as to bring the place of the poles to $c$, still the effects were produced, and apparently with as much power as at first.

94. When the conductor was held against the edge as if fixed to it, and with it moved between the poles, even though but for a few degrees, the galvanometer needle moved and indicated a current of electricity, the same as that which would have been produced if the wheel had revolved in the same direction, the conductor remaining stationary.

95. When the galvanometer connexion with the axis was broken, and its wires made fast to two conductors, both applied to the edge of the copper disc, then currents of electricity were produced, presenting more complicated appearances, but in perfect harmony with the above results. 'Thus, if applied as in fig. 11, a current of electricity through the galvanometer was produced; but if their place was a little shifted, as in fig. 12, a current in the contrary direction resulted; the fact being, that in the first instance the galvanometer indicated the difference between a strong current through $\mathbf{A}$ and a weak one through $\mathbf{B}$, and in the second, of a weak current through $\mathbf{A}$ and a strong one through $\mathbf{B}$ (92.), and therefore produced opposite deflections.

96. So also when the two conductors were equidistant from the magnetic poles, as in fig. 13, no current at the galvanometer was perceived, whichever way the disc was rotated, beyond what was momentarily produced by irregularity of contact; because equal currents in the same direction tended to pass into both. But when the two conductors were connected with one wire, and the axis with the other wire, (fig. 14,) then the galvanometer showed a current according with the direction of rotation (91.) ; both conductors now acting consentaneously, and as a single conductor did before (88.).

97. All these effects could be obtained when only one of the poles of the 
magnet was brought near to the plate; they were of the same kind as to direction, \&c., but by no means so powerful.

98. All care was taken to render these results independent of the earth's magnetism, or of the mutual magnetism of the magnet and galvanometer needles. The contacts were made in the magnetic equator of the plate, and at other parts; the plate was placed horizontally, and the poles vertically; and other precautions were taken. But the absence of any interference of the kind referred to, was readily shown by the want of all effect when the disc was removed from the poles, or the poles from the disc; every other circumstance remaining the same.

99. The relation of the current of electricity produced, to the magnetic pole, to the direction of rotation of the plate, \&c. \&c., may be expressed by saying, that when the unmarked pole (44.84.) is beneath the edge of the plate, and the latter revolves horizontally, screw-fashion, the electricity which can be collected at the edge of the plate nearest to the pole is positive. As the pole of the earth may mentally be considered the unmarked pole, this relation of the rotation, the pole, and the electricity evolved, is not difficult to remember. Or if, in fig. 15, the circle represent the copper disc revolving in the direction of the arrows, and $a$ the outline of the unmarked pole placed beneath the plate, then the electricity collected at $b$ and the neighbouring parts is positive, whilst that collected at the centre $c$ and other parts is negative (88.). The currents in the plate are therefore from the centre by the magnetic poles towards the circumference.

100. If the marked pole be placed above, all other things remaining the same, the electricity at $b$, fig. 15 , is still positive. If the marked pole be placed below, or the unmarked pole above, the electricity is reversed. If the direction of revolution in any case is reversed, the electricity is also reversed.

101. It is now evident that the rotating plate is merely another form of the simpler experiment of passing a piece of metal between the magnetic poles in a rectilinear direction, and that in such cases currents of electricity are produced at right angles to the direction of the motion, and crossing it at the place of the magnetic pole or poles. This was sufficiently shown by the following simple experiment: A piece of copper plate one-fifth of an inch thick, one inch and a half wide, and twelve inches long, being amalgamated at the 
edges, was placed between the magnetic poles, whilst the two conductors from the galvanometer were held in contact with its edges; it was then drawn through between the poles of the conductors in the direction of the arrow, fig. 16; immediately the galvanometer needle was deflected, its north or marked end passed eastward, indicating that the wire A received negative and the wire B positive electricity; and as the marked pole was above, the result is in perfect accordance with the effect obtained by the rotatory plate (99.).

102. On reversing the motion of the plate, the needle at the galvanometer was deflected in the opposite direction, showing an opposite current.

103. To render evident the character of the electrical current existing in various parts of the moving copper plate, differing in their relation to the inducing poles, one collector (86.) only was applied at the part to be examined near to the pole, the other being connected with the end of the plate as the most neutral place; the results are given at fig. 17-20, the marked pole being above the plate. In fig. $17, \mathrm{~B}$ received positive electricity; but the plate moving in the same direction, it received on the opposite side, fig. 18, negative electricity: reversing the motion of the latter, as in fig. 20 , B received positive electricity; or reversing the motion of the first arrangement, that of fig. 17 to fig. 19, B received negative electricity.

104. When the plates were previously removed sideways from between the magnets, as in fig. 21, so as to be quite out of the polar axis, still the same effects were produced, though not so strongly.

105. When the magnetic poles were in contact, and the copper plate was drawn between the conductors near to the place, there was but very little effect produced. When the poles were opened by the width of a card, the effect was somewhat more, but still very small.

106. When an amalgamated copper wire, one eighth of an inch thick, was drawn through between the conductors and poles-(101.), it produced a very considerable effect, though not so much as the plates.

107. If the conductors were held permanently against any particular parts of the copper plates, and carried between the magnetic poles with them, effects the same as those described were produced, in accordance with the results obtained with the revolving disc (94.).

108. On the conductors being held against the ends of the plates, and the 
plates then passed between the magnetic poles, in a direction transverse to their length, the same effects were produced (fig. 22.). The parts of the plates towards the end may be considered either as mere conductors, or as portions of metal in which the electrical current is excited, according to their distance and the strength of the magnet; but the results were in perfect harmony with those before obtained. The effect was as strong as when the conductors were held against the sides of the plate (101.).

109. When the mere wire from the galvanometer, connected so as to form a complete circuit, was passed through between the poles, the galvanometer was affected; and upon passing it to and fro, so as to make the alternate impulses produced correspond with the vibrations of the needle, they could be increased to $20^{\circ}$ or $30^{\circ}$ on each side the magnetic meridian.

110. Upon connecting the ends of a plate of metal with the galvanometer wires, and then carrying it between the poles from end to end, (as in fig. 23.) in either direction, no effect whatever was produced upon the galvanometer. But the moment the motion became transverse, the needle was deflected.

111. These effects were also obtained from electro-magnetic poles, resulting from the use of copper helices or spirals, either alone or with iron cores (34.54.). The directions of the motions were precisely the same; but the action was much greater when the iron cores were used, than without.

112. When a flat spiral was passed through edgeways between the poles, a curious action at the galvanometer resulted; the needle first went strongly one way, but then suddenly stopped, as if it struck against some solid obstacle, and immediately returned. If the spiral were passed through from above downwards, or from below upwards, still the motion of the needle was in the same direction, then suddenly stopped, and then was reversed. But on turning the spiral half-way round, i. e. edge for edge, then the directions of the motions were reversed, but still were suddenly interrupted and inverted as before. This double action depends upon the halves of the spiral (divided by a line passing through its centre perpendicular to the direction of its motion) acting in opposite directions; and the reason why the needle went to the same side, whether the spiral passed by the poles in the one or the other direction, depended upon the circumstance, that upon changing the motion, the direction of the wires in the approaching half of the spiral was changed also. The 
effects, curious as they appear when witnessed, are immediately referable to the action of single wires (40. 109.).

113. Although the experiments with the revolving plate, wires, and plates of metal, were first successfully made with the large magnet belonging to the Royal Society, yet they were all ultimately repeated with a couple of bar magnets two feet long, one inch and a half wide, and half an inch thick; and, by rendering the galvanometer (87.) a little more delicate, with the most striking results. Ferro-electro-magnets, as those of Mold, Henry, \&c. (57.), are very powerful. It is very essential, when making experiments on different substances, that thermo-electric effects (produced by contact of the fingers, \&c.) be avoided, or at least appreciated and accounted for ; they are easily distinguished by their permanency, and their independence of the magnets.

114. The relation which holds between the magnetic pole, the moving wire or metal, and the direction of the current evolved, i. e. the law which governs the evolution of electricity by magneto-electric induction, is very simple, although rather difficult to express. If in fig. 24. PN represent a horizontal wire passing by a marked magnetic pole, so that the direction of its motion shall coincide with the curved line proceeding from below upwards; or if its motion parallel to itself be in a line tangential to the curved line, but in the general direction of the arrows; or if it pass the pole in other directions, but so as to cut the magnetic curves* in the same general direction, or on the same side as they would be cut by the wire if moving along the dotted curved line; - then the current of electricity in the wire is from $\mathrm{P}$ to $\mathrm{N}$. If it be carried in the reverse directions, the electric current will be from $\mathbf{N}$ to $\mathrm{P}$. Or if the wire be in the vertical position, figured $\mathbf{P}^{\prime} \mathbf{N}^{\prime}$, and it be carried in similar directions, coinciding with the dotted horizontal curve so far, as to cut the magnetic curves on the same side with it, the current will be from $\mathbf{P}^{\prime}$ to $\mathbf{N}^{\prime}$. If the wire be considered a tangent to the curved surface of the cylindrical magnet, and it be carried round that surface into any other position, or if the magnet itself be revolved on its axis, so as to bring any part opposite to the tangential wire,-still, if afterwards the wire be moved in the directions indi-

* By magnetic curves, I mean the lines of magnetic forces, however modified by the juxtaposition of poles, which would be depicted by iron filings; or those to which a very small magnetic needle would form a tangent. 
cated, the current of electricity will be from $\mathbf{P}$ to $\mathbf{N}$; or if it be moved in the opposite direction, from $\mathbf{N}$ to $\mathbf{P}$; so that as regards the motions of the wire past the pole, they may be reduced to two, directly opposite to each other, one of which produces a current from $\mathbf{P}$ to $\mathbf{N}$, and the other from $\mathbf{N}$ to $\mathbf{P}$.

115. The same holds true of the unmarked pole of the magnet, except that if it be substituted for the one in the figure, then, as the wires are moved in the direction of the arrows, the current of electricity would be from $N$ to $P$, and as they move in the reverse direction, from $\mathbf{P}$ to $\mathbf{N}$.

116. Hence the current of electricity which is excited in metal when moving in the neighbourhood of a magnet, depends for its direction altogether upon the relation of the metal to the resultant of magnetic action, or to the magnetic curves, and may be expressed in a popular way thus ; Let A B (fig. 25.) represent a cylinder magnet, $A$ being the marked pole, and $B$ the unmarked pole; let $\mathrm{PN}$ be a silver knife-blade resting across the magnet with its edge upward, and with its marked or notched side towards the pole $A$; then in whatever direction or position this knife be moved edge foremost, either about the marked or the unmarked pole, the current of electricity produced will be from $\mathrm{P}$ to $\mathrm{N}$, provided the intersected curves proceeding from $\mathrm{A}$ abut upon the notched surface of the knife, and those from B upon the unnotched side. Or if the knife be moved with its back foremost, the current will be from $\mathbf{N}$ to $P$ in every possible position and direction, provided the intersected curves abut on the same surfaces as before. A little model is easily constructed, by using a cylinder of wood for a magnet, a flat piece for the blade, and a piece of thread connecting one end of the cylinder with the other, and passing through a hole in the blade, for the magnetic curves: this readily gives the result of any possible direction.

117. When the wire under induction is passing by an electro-magnetic pole, as for instance one end of a copper helix traversed by the electric current(34.), the direction of the current in the approaching wire is the same with that of the current in the parts or sides of the spirals nearest to it, and in the receding wire the reverse of that in the parts nearest to it.

118. All these results show that the power of inducing electric currents is circumferentially excited by a magnetic resultant or axis of power, just as circumferential magnetism is dependent upon and is exhibited by an electric current. 
119. The experiments described combine to prove that when a piece of metal (and the same may be true of all conducting matter) is passed either before a single pole; or between the opposite poles of a magnet, or near electromagnetic poles, whether ferruginous or not, electrical currents are produced across the metal transverse to the direction of motion; and which therefore, in Arago's experiments, will approximate towards the direction of radii. If a single wire be moved like the spoke of a wheel near a magnetic pole, a current of electricity is determined through it from one end towards the other. If a wheel be imagined, constructed of a great number of these radii, and this revolved near the pole, in the manner of the copper disc (85.), each radius will have a current produced in it as it passes by the pole. If the radii be supposed to be in contact laterally, a copper disc results, in which the directions of the currents will be generally the same, being modified only by the coaction which ean take place between the particles, now that they are in metallic contact.

120. Now that the existence of these currents is known, Arago's phenomena may be accounted for without considering them as due to the formation in the copper of a pole of the opposite kind to that approximated, surrounded by a diffuse polarity of the same kind (82.); neither is it essential that the plate should acquire and lose its state in a finite time; nor on the other hand does it seem necessary that any repulsive force should be admitted as the cause of the rotation (82.).

121. The effect is precisely of the same kind as the electro-magnetic rotations which I had the good fortune to discover some years ago*. According to the experiments then made, which have since been abundantly confirmed, if a wire (PN, fig. 26.) be connected with the positive and negative ends of a voltaic battery, so that the positive electricity shall pass from $P$ to $N$, and a marked magnetic pole $\mathrm{N}$ be placed near the wire between it and the spectator, the pole will move in a direction tangential to the wire, i. e. towards the right, and the wire will move tangentially towards the left, according to the directions of the arrows. This is exactly what takes place in the rotation of a plate beneath a magnetic pole; for let N (fig. 27.) be a marked pole above the circular plate, the latter being rotated in the direction of the arrow: immediately

\footnotetext{
* Quarterly Journal of Science, vol. xii. pp. 74. 186, 416. 283.
} 
currents of positive electricity set from the central parts in the general direction of the radii by the pole to the parts of the circumference $a$ on the other side of that pole (99. 119.), and are therefore exactly in the same relation to it as the current in the wire (PN, fig. 26.) and therefore the pole in the same manner moves to the right hand.

122. If the rotation of the disc be reversed, the electric currents are reversed (91.), and the pole therefore moves to the left hand. If the contrary pole be employed, the effects are the same, i. e. in the same direction, because currents of electricity, the reverse of those described, are produced, and by reversing both poles and currents, the visible effects remain unchanged. In whatever position the magnetic axis be placed, provided the same pole be applied to the same side of the plate, the electric current produced is in the same direction, in consistency with the law already stated (114, \&c.); and thus every circumstance regarding the direction of the motion may be explained.

123. These currents are discharged or return in the parts of the plate on each side of and more distant from the place of the pole, where, of course, the magnetic induction is weaker: and when the collecters are applied, and a current of electricity is carried away to the galvanometer, the deflection there is merely a repetition, by the same current or part of it, of the effect of rotation in the magnet over the plate itself.

124. It is under the point of view just put forth that $I$ have ventured to say it is not necessary that the plate should acquire and lose its state in a finite time (120.); for if it were possible for the current to be fully developed the instant before it arrived at its state of nearest approximation to the vertical pole of the magnet, instead of opposite to or a little beyond it, still the relative motion of the pole and plate would be the same, the resulting force being tangential instead of direct.

125. But it is possible (though not necessary for the rotation) that time may be required for the development of the maximum current in the plate, in which case the resultant of all the forces would be in advance of the magnet when the plate is rotated, or in the rear of the magnet when the latter is rotated, and many of the effects with pure electro-magnetic poles tend to prove this is the case. Then, the tangential force may be resolved into two others, one parallel to the plane of rotation, and the other perpendicular to it; the 
former would be the force excited in making the plate. revolve with the magnet, or the magnet with the plate; the latter would be a repulsive force, and is probably that, the effects of which M. Arago has discovered (82.).

126. The extraordinary circumstance accompanying this action, which has seemed so inexplicable, namely, the cessation of all phenomena when the magnet and metal are brought to rest, now receives a full explanation (82.); for then the electrical currents which cause the motion, cease altogether.

127. All the effects of solution of metallic continuity, and the consequent diminution of power described by Messrs. Babbage and Herschel*, now receive their natural explanation, as well also as the resumption of power when the cuts were filled up by metallic substances, which, though conductors of electricity, were themselves very deficient in the power of influencing magnets. And new modes of cutting the plate may be devised, which shall almost entirely destroy its power. Thus, if a copper plate (81.) be cut through at about a fifth or sixth of its diameter from the edge, so as to separate a ring from it, and this ring be again fastened on, but with a thickness of paper intervening (fig. 29.), and if AraGo's experiment be made with this compound plate so adjusted that the section shall continually traverse opposite the pole, it is evident that the magnetic currents will be greatly interfered with, and the plate probably lose much of its effect $\gamma$.

An elementary result of this kind was obtained by using two pieces of thick copper, shaped as in fig. 28. When the two neighbouring edges were amalgamated and put together, and the arrangement passed between the poles of the magnet, in a direction parallel to these edges, a current was urged through the wires attached to the outer angles, and the galvanometer became strongly affected; but when a single film of paper was interposed, and the experiment repeated, no sensible effect could be produced.

128. A section of this kind could not interfere much with the induction of magnetism, supposed to be of the nature ordinarily received by iron.

129. The effect of rotation or deflection of the needle, which M. Arago obtained by ordinary magnets, M. AMpere succeeded in procuring by electro-

* Philosophical Transactions, 1825, p. 481.

+ This experiment has actually been made by Mr. CHristie, with the results here described, and is recorded in the Philosophical Transactions for 1827. p. 82. 
magnets. This is perfectly in harmony with the results relative to voltaelectric and magneto-electric induction described in this paper. And by using flat spirals of copper wire, through which electric currents were sent, in place of ordinary magnetic poles (111.), sometimes applying a single one to one side of the rotating plate, and sometimes two to opposite sides, I obtained the induced currents of electricity from the plate itself, and could lead thein away to, and ascertain their existence by, the galvanometer.

130. The cause which has now been assigned for the rotation in Arago's experiment, namely, the production of electrical currents, seems abundantly sufficient in all cases where the metals, or perhaps even other conductors, are concerned; but with regard to such bodies as glass, resins and, above all, gases, it seems impossible that currents of electricity capable of producing these effects should be generated in them. Yet Arago found that the effects in question were produced by these and by all bodies tried (81.). Messrs. BABbaGE and Hersches, it is true, did not observe them with any substance not metallic, except carbon, in a highly conducting state (82.). Mr. Harris has ascertained their occurrence with wood, marble, freestone and annealed glass, but obtained no effect with sulphuric acid and saturated solution of sulphate of iron, although these are better conductors of electricity than the former substances.

131. Future investigations will no doubt explain these difficulties, and decide the point whether the retarding or dragging action spoken of is always simultaneous with electric currents *. The existence of the action in metals, only whilst the currents exist, i. e. whilst motion is given (82. 88.), and the explication of the repulsive action observed by M. Arago (82. 125), are the strong reasons for referring it to this cause; but it may be combined with others which occasionally act alone.

132. Copper, iron, tin, zinc, lead, mercury, and all the metals tried, produced electrical currents when passed between the magnetic poles: the mercury was put into a glass tube for the purpose. The dense carbon deposited in

* Experiments which I have since made convince me that this particular action is always due to the electrical currents formed; and they supply a test by which it may be distinguished from the action of ordinary magnetism, or any other cause, including those which are mechanical or irregular, producing similar effects. 
coal gas retorts, also produced the current, but ordinary charcoal did not. Neither could I obtain any sensible effects with brine, sulphuric acid, saline solutions, \&c., whether rotated in basins, or inclosed in tubes and passed between the poles.

133. I have never been able to produce any sensation upon the tongue by the wires connected with the conductors applied to the edges of the revolving plate (88.) or slips of metal (101.). Nor have I been able to heat a fine platina wire, or produce a spark, or convulse the limbs of a frog. I have failed also to produce any chemical effects by electricity thus evolved (22. 56.).

134. As the electric current in the revolving copper plate occupies but a small space, proceeding by the poles and being discharged right and left at very small distances comparatively; and as it exists in a thick mass of metal possessing almost the highest conducting power of any, and consequently offering extraordinary facility for its production and discharge; and as, notwithstanding this, considerable currents may be drawn off which can pass through narrow wires, forty, fifty, sixty, or even one hundred feet long; it is evident that the current existing in the plate itself must be a very powerful one, when the rotation is rapid and the magnet strong. This is also abundantly proved by the obedience and readiness with which a magnet ten or twelve pounds in weight follows the motion of the plate and will strongly twist up the cord by which it is suspended.

135. Two rough trials were made with the intention of constructing magneto-electric machines. In one, a ring one inch and a half broad and twelve inches external diameter, cut from a thick copper plate, was mounted so as to revolve between the poles of the magnet and represent a plate similar to those formerly used (101.), but of interminable length; the inner and outer edges were amalgamated, and the conductors applied one to each edge, at the place of the magnetic poles. The current of electricity evolved did not appear by the galvanometer to be stronger, if so strong, as that from the circular plate (88.).

136. In the other, small thick discs of copper or other metal, half an inch in diameter, were revolved rapidly near to the poles, but with the axis of rotation out of the polar axis; the electricity evolved was collected by conductors applied as before to the edges (86.). Currents were procured, but of strength much inferior to that produced by the circular plate. 
137. The latter experiment is analogous to those made by Mr. BarLow with a rotating iron shell, subject to the infiuence of the earth*. The effects then obtained have been referred by Messrs. Babbage and Herschel to the same cause as that considered as influential in Arago's experiment $\gamma ;$; but it would be interesting to know how far the electric current which might be produced in the experiment would account for the deflexion of the needle. The mere inversion of a copper wire six or seven times near the poles of the magnet, and isochronously with the vibrations of the galvanometer needle connected with it, was sufficient to make the needle vibrate through an arc of $60^{\circ}$ or $70^{\circ}$. The rotation of a copper shell would perhaps decide the point, and might even throw light upon the more permanent, though somewhat analogous effects obtained by Mr. Christie.

138. The remark which has already been made respecting iron (66.), and the independence of the ordinary magnetical phenomena of that substance, and the phenomena now described of magneto-electric induction in that and other metals, was fully confirmed by many results of the kind detailed in this section. When an iron plate similar to the copper one formerly described (101.) was passed between the magnetic poles, it gave a current of electricity like the copper plate, but decidedly of less power; and in the experiments upon the induction of electric currents (9.), no difference in the kind of action between iron and other metals could be perceived. The power therefore of an iron plate to drag a magnet after it, or to intercept magnetic action, should be carefully distinguished from the similar power of such metals as silver, copper, \&c. \&c. inasmuch as in the iron by far the greater part of the effect is due to what may be called ordinary magnetic action. There can be no doubt that the cause assigned by Messrs. Babbage and Herscher in explication of Arago's phenomena is true when iron is the metal used.

139. The very feeble powers which were found by those philosophers to belong to bismuth and antimony, when moving, of affecting the suspended magnet, and which has been confirmed by Mr. HArris, seem at first disproportionate to their conducting powers ; whether it be so or not must be decided by future experiment (73.) $\star$. These metals are highly crystalline, and probably conduct

* Philosophical Transactions, 1825. p. 317.

† Ibid. 1825. p. 485.

$¥$ I have since been able to explain these differences, and prove, with several metals, that the effect is in the orler of the conducting power; for I have been able to obtain, by magneto-electric induction, MDCCCXXXII. 
162 MR. FARADAY'S EXPERIMENTAL RESEARCHES IN ELECTRICITY.

electricity with different degrees of facility in different directions; and it is not unlikely that where a mass is made up of a number of crystals heterogeneously associated, an effect approaching to that of actual division may occur (127.); or the currents of electricity may become more suddenly deflected at the confines of similar crystalline arrangements, and so be more readily and completely discharged within the mass.

currents of electricity which are proportionate in strength to the conducting power of the bodies experimented with (211.).

Royal Institution, November 1831.

Note.-In consequence of the long period which has intervened between the reading and printing of the foregoing paper, accounts of the experiments have been dispersed, and, through a letter of my own to M. HAchetre, have reached France and Italy. That letter was translated (with some errors), and read to the Academy of Sciences at Paris, 26th December, 1831. A copy of it in Le Temps of the 28th December quickly reached Signor Noвili, who, with Signor Antinori, immediately experimented upon the subject, and obtained many of the results mentioned in my letter; others they could not obtain or understand, because of the brevity of my account. These results by Signori NoBILI and ANTINon have been embodied in a paper dated 31st January 1832, and printed and published in the number of the Antologia dated November 1831, (according at least to the copy of the paper kindly sent me by Signor NoBILI). It is evident the work could not have been then printed; and though Signor Noвil, in his paper, has inserted my letter as the text of his experiments, yet the circumstance of back date has caused many here, who have heard of NoBILr's experiments by report only, to imagine his results were anterior to, instead of being dependent upon, mine.

I may be allowed under these circumstances to remark, that I experimented on this subject several years ago, and have published results. (See Quarterly Journal of Science for July 1825. p. 338.) The following also is an extract from my note-book, dated November 28, 1825 : " Experiments on induction by connecting wire of voltaic battery :-a battery of four troughs, ten pairs of plates, each arranged side by side-the poles connected by a wire about four feet long, parallel to which was another similar wire separated from it only by two thicknesses of paper, the ends of the latter were attached to a galvanometer :-exhihited no action, \&c. \&c. \&c.-Could not in any way render any induction evident from the connecting wire." The cause of failure at that time is now evident (79.).-M. F. April 1832. 



\begin{tabular}{|c|c|c|c|}
\hline & & & \\
\hline
\end{tabular}

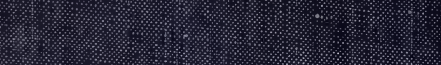

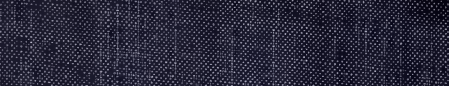

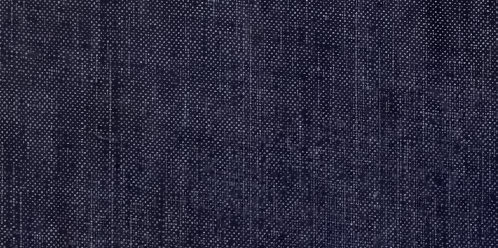

\begin{tabular}{|l|l}
1 \\
1
\end{tabular}

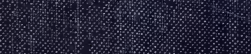

1
1

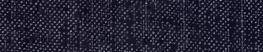

1.:

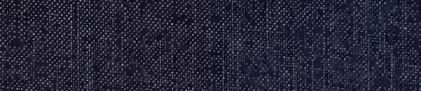

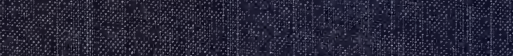

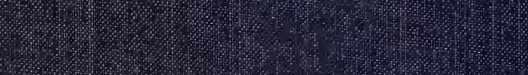

\begin{tabular}{|c|c|c|c|}
\hline \\
\hline
\end{tabular}

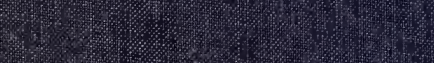

0
3

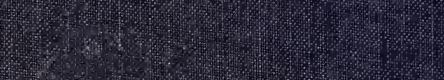

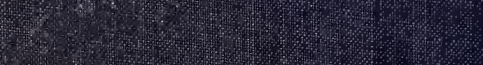

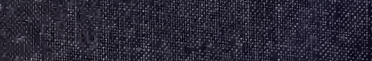

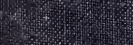

(2) 